\title{
TOUCHING ON THE EFFECTS OF AN IMPERFECT WINDOW FUNCTION
}

W. J. CHAPLIN. Y. ELSWORTH. G. R. ISAAK. C. P. MCLEOD AND B. A. MILLER

School of Physics \& Astronomy, University of Birmingham

Edgbaston, Birmingham B15 2TT UK

E-mail:wjc@star.sr.bham.ac.uk

AND

R. NEW

School of Science Eं Mathematics, Sheffield Hallam University Sheffield, S1 $1 \mathrm{WB}$

BiSON currently consists of four fully- and two semi-automated observatories, dedicated to the round-the-clock collection of low-degree (low- $\ell$ ) helioseismological data. A complete historical record of the network can be found in Chaplin et al. (1996). The annual duty cycles achieved by BiSON have improved steadily since our sixth site was commissioned in 1992. Over the period 1992 - 1996 inclusive. we have recorded annual duty cycles of: $54,68,78,74$ and 78 per cent respectively.

While a quasi-diurnal gap structure persists in combined, 6-station BiSON time series, giving rise to weak sidebands in the frequency domain. they also contain a large number of short gaps. We are currently investigating the feasibility of filling short gaps with estimates of the solar Doppler velocity signal, in an effort to reduce the contamination introduced by the effects of breaks in the data stream. In order to illustrate the impact of an imperfect window function. we have performed a series of simulations with artificial $p$-mode data.

The plots in Fig. 1 show the effect of imposing a 4-month BiSON window function (1996 Mar 27 - 1996 July 26) on a set of artificial modes generated in the time domain. Some 101 simulated Doppler velocity mode residuals - mapping the range $0 \leq n \leq 3$ and $1185 \leq \nu \leq 4665 \mu \mathrm{Hz}$ - were produced by a model based upon a stochastically forced, damped harmonic oscillator (for full details see Chaplin et al. 1997). The mode frequencies, relative powers and lifetimes were selected in order to reflect the characteristics of the observed, low-degree solar $p$-mode spectrum. The 4-month window function was then applied to the resulting time series, and the power spectrum computed. We also computed: the spectrum from a 100 -per-cent fill time series (i.e.. no window function applied): and a spectrum where the 4-month window function was applied, but where gaps of duration $1 \mathrm{hr}$ or less were "removed" (i.e., data were restored to the time series. increasing the fill by $\approx 4$ per cent). We have also included a plot of the actual BiSON low-degree spectrum for this period. 

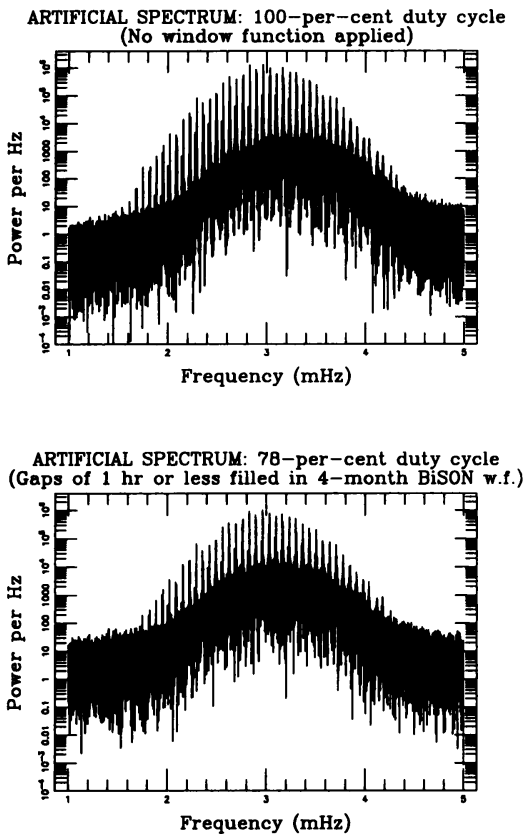
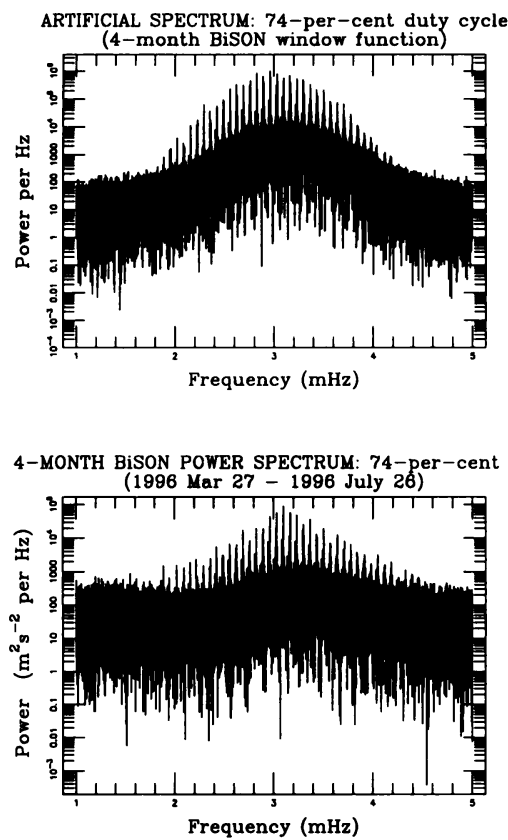

Figure 1. These plots show the results of applying a 4-month BiSON window function from 1996 to a time series of artificial $p$-mode residuals (see text). Also included is a plot of the actual BiSON low-degree spectrum from this period.

The results are rather striking: the window function gives rise to a factor-of-ten reduction in the signal to noise near the centre of the spectrum. The large increase in the background level also impacts upon the detectability of modes at the extreme ends of the spectrum. By "filling" short gaps in the time series, the deterioration in quality is greatly reduced. Provided one were able to fill such gaps with reliable estimates of the true. solar data, these simulations imply that the resulting gains in signal to noise would be quite marked for ground-based networks like BiSON. This is clearly of most importance to those modes with low signal-to-noise ratios - the resulting improvement in the measured precision of the frequencies of the strongest modes would be quite modest.

\section{References}

Chaplin W. J., Elsworth Y., Howe R., Isaak G. R., McLeod C. P., Miller B. A., New R., van der Raay H. B. and Wheeler S. J., Solar Physics, 1996, 168, 1

Chaplin W. J., Elsworth Y., Howe R., Isaak G. R., McLeod C. P., Miller B. A. and New R., 1997, MNRAS, 1997, 287, 51 